\title{
Disposable sample holder for high temperature measurements in MPMS superconducting quantum interference device magnetometers
}

\author{
J. Sesé \\ Instituto Universitario de Investigación en Nanociencia de Aragón, Universidad de Zaragoza, \\ C/Pedro Cerbuna 12, 50009 Zaragoza, Spain \\ J. Bartolomé and C. Rillo \\ Instituto de Ciencia de Materiales de Aragón, CSIC-Universidad de Zaragoza, \\ C/Pedro Cerbuna 12, 50009 Zaragoza, Spain
}

(Received 10 January 2007; accepted 4 March 2007; published online 2 April 2007)

\begin{abstract}
A sample holder for high temperature $(300 \mathrm{~K}<T<800 \mathrm{~K})$ measurements in superconducting quantum interference device magnetometers is presented. It is fabricated using aluminum foil and it is appropriate for samples in either solid or powder form. The holder is homogeneous for the gradiometer coil, and this results in a contribution to the background signal that is below the instrument noise at any field $\left(<10^{-9} \mathrm{~A} \mathrm{~m}^{2}\right.$ at $\left.\mu_{0} H=200 \mathrm{mT}\right)$. Further it is inexpensive and simple to fabricate, and it can be considered as a disposable sample holder that avoids eventual contamination between different samples. (C) 2007 American Institute of Physics.
\end{abstract}

[DOI: $10.1063 / 1.2720722$ ]

The superconducting quantum interference device (SQUID) is a very sensitive magnetic flux detector. Nowadays, SQUID-based magnetometers are commercially available with a high degree of automation and a friendly use for nonspecialists. One successful example is the MPMS system from Quantum Design, Inc. ${ }^{1}$ The use of these instruments has spread in a wide variety of scientific areas in which magnetism plays a significant role.

The sample space in MPMS system is cylindrical with a diameter of $9 \mathrm{~mm}$, but the sample size should be smaller to be considered as a point dipole. It has been calculated that this condition holds when the sample volume does not exceed $5 \%$ of the total gradiometer volume. ${ }^{2}$ The second order gradiometer is composed of coils with $2 \mathrm{~cm}$ diameter and separated by a total distance $3 \mathrm{~cm}$, resulting in a volume of $9.4 \mathrm{~cm}^{3}$.

A characteristic of the standard MPMS system $(1.8 \mathrm{~K}<T<400 \mathrm{~K})$ is the simple sample holder. Typical sample dimensions are $5 \times 5 \times 5 \mathrm{~mm}^{3}$ and it is easily mounted inside a disposable plastic straw that is laterally attached to the sample rod and inserted in the instrument.

However, when the oven option of the MPMS systems is installed $(300 \mathrm{~K}<T<800 \mathrm{~K})$, the available sample space is considerably reduced to $3.5 \mathrm{~mm}$ diameter. The high working temperature and the reduced sample space have made elusive finding a simple sample holder like the one used in the standard MPMS. Indeed, despite the number of MPMS systems available, there are few high temperature SQUID measurements found in the literature. Recently, a specially fabricated quartz sample holder that can be particularly useful for thin films ${ }^{3}$ has been described, but it produces a background signal and cannot be used for samples of arbitrary shape.

Here, a disposable sample holder for the oven option in MPMS systems that can be considered analogous to the plastic straw used in the standard MPMS is presented.
The sample holder presented here fulfils the criteria previously stated ${ }^{3}$ to be suitable for magnetic measurements at elevated temperatures. Namely, it is mechanically and thermally stable until $800 \mathrm{~K}$, its contribution to the background signal is below the instrument noise, and the outer diameter does not exceed $3.2 \mathrm{~mm}$. In addition, the implementation is very simple and inexpensive; thus, it results in a disposable sample holder that avoids contamination between samples.

The fabrication of the sample holder is illustrated in Fig. 1. It is made using aluminum foil with thickness of $10 \mu \mathrm{m}$. A rectangular piece of approximately $1 \times 15 \mathrm{~cm}^{2}$ is cut, and the sample is located approximately $4 \mathrm{~cm}$ from one end. Then the foil is rolled up around the sample and around itself resulting in a thin cylinder that is quite rigid and easy to handle (see Fig. 1). It can then be fixed to the sample rod by making a small hook and using a short length of copper wire as shown in Figs. 1 and 2.

We have compared the magnetic properties of a regular aluminum foil for domestic use with an aluminum foil from

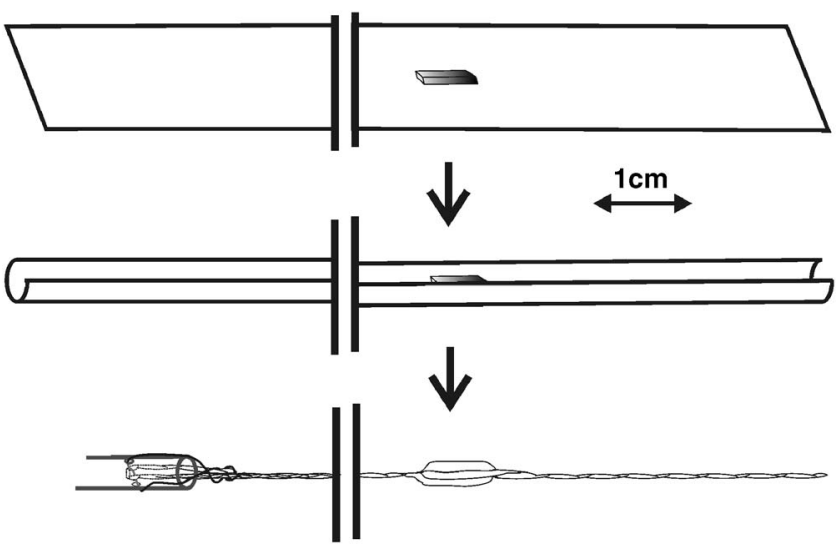

FIG. 1. The sample is placed in the aluminum foil and rolled up to form a thin cylinder that is easily attached to the sample rod. 


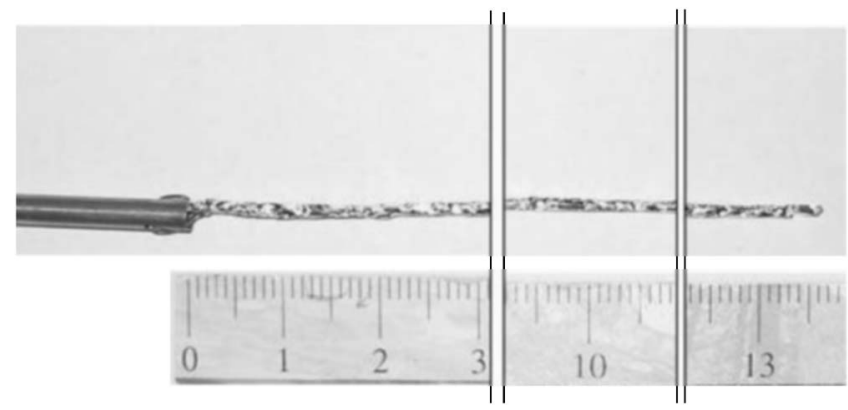

FIG. 2. Photograph of the sample holder with the nickel sample inside.

a reference source ${ }^{4}$ (purity of $99 \%$, thickness of $0.01 \mathrm{~mm}$, and coil width of $300 \mathrm{~mm}$ ). The magnetic susceptibility at $300 \mathrm{~K}$ and magnetic fields up to $1.6 \times 10^{7} \mathrm{~A} / \mathrm{m}$ was measured to be $(1.97 \pm 0.04) \times 10^{-5}$ and $(2.00 \pm 0.04) \times 10^{-5}$, respectively. These values are in good agreement with published results for pure aluminum, $2.04 \times 10^{-5} .{ }^{5}$ In both cases, we also measured a comparatively small additional ferromagnetic signal that saturates above $2.4 \times 10^{5} \mathrm{~A} / \mathrm{m}$ and has a value of 0.54 and $1.1 \mathrm{~A} / \mathrm{m}$, respectively.

A laboratory oven was used to check the thermal stability of the aluminum sample holder for temperatures up to $850 \mathrm{~K}$. No changes were appreciated despite the relative proximity to the melting point of aluminum, $933 \mathrm{~K}$. The thermal expansion coefficient of aluminum is $23 \times 10^{-6} \mathrm{deg}^{-1}$. For a $300-800 \mathrm{~K}$ sweep, this represents a maximum movement of the sample of approximately $1 \mathrm{~mm}$. This displacement is automatically corrected when the iterative fitting algorithm of the instrument is used. Alternatively, the sample position can be automatically centered at intermediate temperatures.

Previous designs of sample holder have a background magnetic contribution due to the empty sample space not occupied by the holder. The sample holder here presented has an intrinsic homogeneous distribution of aluminum along the sample holder, and thus there is no uncompensated empty space. In consequence, measurements performed with an empty sample holder give values of magnetic moment below $10^{-9} \mathrm{~A} \mathrm{~m}^{2}$ that are due to the instrument noise. Same results were obtained for the two different aluminum foils previously described.

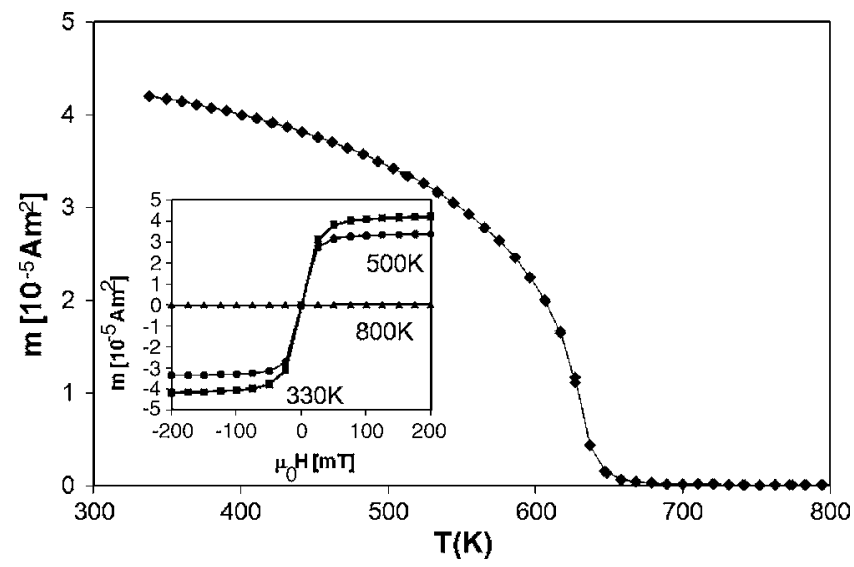

FIG. 3. Magnetic moment vs temperature measurements for the nickel sample using $\mu_{0} H_{\mathrm{dc}}=200 \mathrm{mT}$. The inset shows $m(H)$ curves at different temperatures.

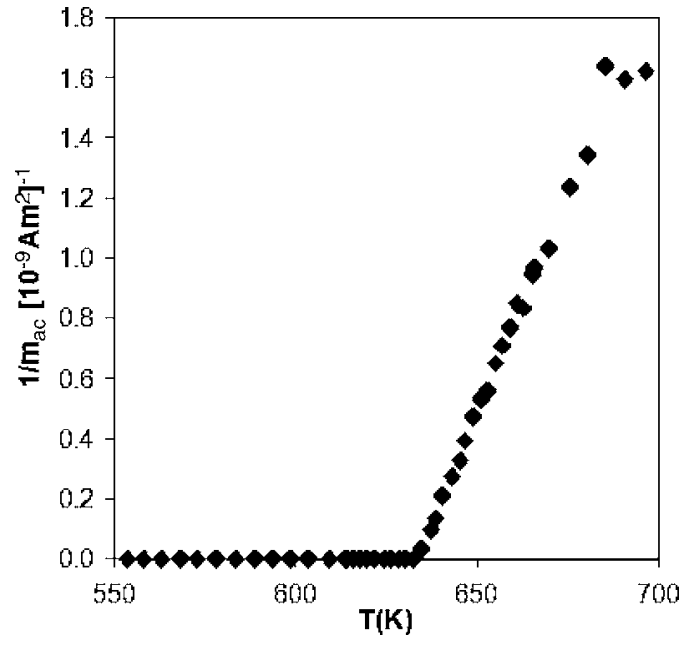

FIG. 4. Inverse of ac magnetic moment vs temperature for the nickel sample measured with $\mu_{0} H_{\mathrm{ac}}=0.4 \mathrm{mT}, f=10 \mathrm{~Hz}$, and $\mu_{0} H_{\mathrm{dc}}=0$.

With this new holder, the sample size can approach the maximum available sample space diameter because the small thickness of the foil has little impact on the total sample volume. Even more, samples in small pieces or powder form are very suitable for this holder.

We have performed $m(T), m(H)$, and $m_{\mathrm{ac}}(T)$ measurements on a nickel bulk metallic sample and a cobalt thin film to evaluate the response of the system and the sample to holder signal ratio. We used $4 \mathrm{~cm}$ long scans with the SQUID amplifier in autorange and default filter settings.

The nickel sample is composed of several small pieces with a total mass of $0.88 \mathrm{mg}$ (nickel slug $99.995 \%$ Puratronic ${ }^{\circledR}$ from Alfa Aesar). ${ }^{6}$ The transition from ferromagnetic to paramagnetic is clearly seen in Figs. 3 and 4 in which the Curie temperature can be determined. Figure 3 shows a $m(T)$ measurement with $\mu_{0} H_{\mathrm{dc}}=200 \mathrm{mT}$ and $m(H)$ curves for $T=330,500$, and $800 \mathrm{~K}$. Figure 4 shows the magnetization response amplitude, $m_{\mathrm{ac}}(T)$ to a sinusoidal magnetic field excitation with amplitude $\mu_{0} H_{\mathrm{ac}}=0.4 \mathrm{mT}, f=10 \mathrm{~Hz}$, and $\mu_{0} H_{\mathrm{dc}}=0$; from this figure, the Curie temperature is esti-

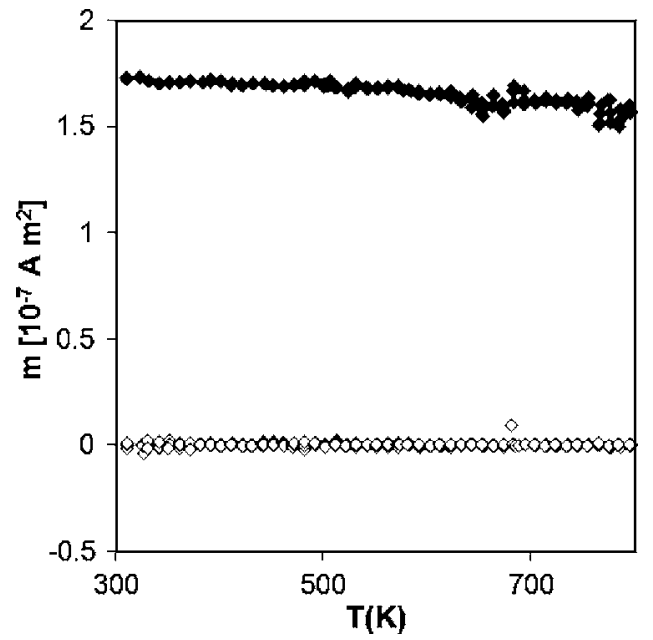

FIG. 5. Magnetic moment vs temperature measurement of the cobalt thin film sample. Open symbols correspond to the measured signal when the sample is removed from the sample holder; the average value is 3 $\times 10^{-10} \mathrm{~A} \mathrm{~m}^{2}$ with a standard deviation of $9 \times 10^{-10} \mathrm{~A} \mathrm{~m}^{2}$. 


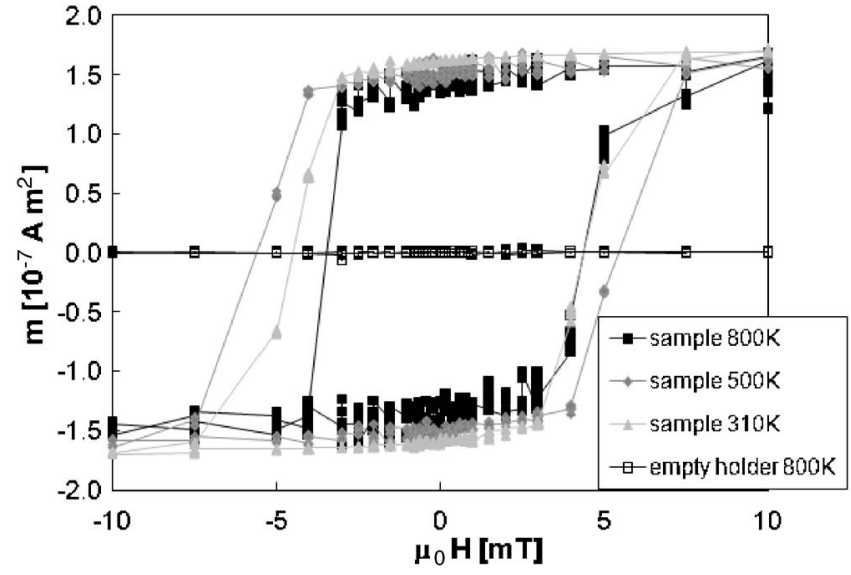

FIG. 6. Magnetic moment vs magnetic field curves of the cobalt thin film with different temperatures $T=310,500$, and $800 \mathrm{~K}$. Open symbols correspond to the measured signal at $T=800 \mathrm{~K}$ when the sample is removed from the sample holder at $T=800 \mathrm{~K}$; the average value is $4 \times 10^{-11} \mathrm{~A} \mathrm{~m}^{2}$ with a standard deviation of $7 \times 10^{-10} \mathrm{~A} \mathrm{~m}^{2}$.

mated to be $633 \pm 1 \mathrm{~K}$, which is in accordance with values reported in literature, $631-636 \mathrm{~K} .^{5}$

The cobalt thin film is $15 \mathrm{~nm}$ thick and was sputter deposited on a silicon substrate with an approximate area of $5 \times 1.5 \mathrm{~mm}^{2}$. This sample was used to compare a smallsignal sample with the signal coming from the empty holder. For that purpose, the measurements were performed first with the sample placed on the holder, the sample was then removed from the holder, and the measurement was repeated with the same centering position. Figure 5 shows a $m(T)$ measurement with $\mu_{0} H_{\mathrm{dc}}=10 \mathrm{mT}$, and Fig. 6 shows $m(H)$ curves with $T=310,500$, and $800 \mathrm{~K}$. In all cases, the signal coming from the holder is negligible compared to the sample signal.

A disposable sample holder for high temperature measurements using the oven option in MPMS instruments has been presented. The holder is designed to give a negligible background contribution and has been tested in $m(T), m(H)$, and $m_{\mathrm{ac}}(T)$ measurements up to $800 \mathrm{~K}$. The difficulties in performing this type of measurements are now considerably reduced.

The authors would like to thank the Spanish Ministry of Education and Science through the "Ramon y Cajal" Program, Project No. MAT 2005/01272 and project "Renovación y ampliación del Servicio de Medidas Magnéticas," University of Zaragoza-CSIC (PAI 2004), for the financial support.

\footnotetext{
${ }^{1}$ Quantum Design, Inc., 11578 Sorrento Valley Road, San Diego, CA (www.qdusa.com).

${ }^{2}$ P. Stamenov and J. M. D. Coey, Rev. Sci. Instrum. 77, 015106 (2006).

${ }^{3}$ S. Bedanta, O. Petracic, M. Aderholz, and W. Kleemann, Rev. Sci. Instrum. 76, 083910 (2005).

${ }^{4}$ Reference AL000370, www.goodfellow.com

${ }^{5}$ Zahlenwerte und Funktionen, Landolt-Börnstein, 6th edition, edited by Vogt and Höhl (Springer, Berlin, 1962), Vol. 2, Pt. 9.

${ }^{6}$ www.alfa-chemcat.com
} 